\title{
Desenvolvimento de um analisador de incubadoras neonatais
}

\author{
Josemir da Cruz Alexandrino*, Handerson Jorge Dourado Leite, Ricardo de Araújo Kalid, \\ Alexandre Henrique Hermini, Herman Augusto Lepikson
}

Resumo Embora tenha sido concebida para beneficiar recém-nascidos $(\mathrm{RN})$ prematuros, pois cria um ambiente artificial próximo das condições uterinas, a incubadora neonatal pode expor o $\mathrm{RN}$ a várias fontes de risco. Inspeções periódicas para verificação de requisitos mínimos de desempenho e segurança podem identificar tais fontes de risco e seus graus de incidência, contribuindo para minimizá-los. Visando a automação de ensaios de avaliação metrológica deste tipo de equipamento médico (EM), apresenta-se o desenvolvimento de um analisador, com capacidade de efetuar todas as medições das variáveis definidas na norma NBR IEC 60601-2-19 e de se comunicar com um computador, permitindo a transmissão dos dados de medição e recepção de comandos para a realização de ajustes remotos. O desenvolvimento desse sistema cria uma alternativa nacional, de menor custo e com características construtivas mais adequadas aos ensaios metrológicos requeridos. Como resultado obteve-se um protótipo microcontrolado, modular, portátil e de fácil uso que realiza a medição das variáveis: temperatura, umidade relativa, concentração de oxigênio, ruído e velocidade do ar. Dados das calibrações demonstram que o erro máximo relativo e a incerteza da medição para os subsistemas de temperatura, umidade relativa, nível de ruído e concentração de $\mathrm{O}_{2}$ foram $\left(-0,42 ; 0,05^{\circ} \mathrm{C}\right),( \pm 0,72 ; 0,24 \% \mathrm{UR})$, $(-5,17 ; 0,68 \mathrm{~dB})$ e $(1,28 ; 2,2 \%)$ respectivamente. Tais resultados indicam que o analisador desenvolvido apresenta uma qualidade metrológica compatível com os requisitos normativos estabelecidos.

Palavras-chave: Avaliação de equipamentos médicos, Metrologia, Engenharia clínica.

\section{Development of a neonatal incubator analyzer}

Abstract Although designed to benefit premature newborns (NB) by creating an artificial environment close to the uterine
conditions, the use of neonatal incubators may expose the NB to several sources of risk. Periodic inspections
for verification of minimum requirements of performance and safety can identify these and their degrees
of impact, helping to minimize them. In order to automate the metrological evaluation essays in neonatal
incubators, we present the development of an analyzer with the capacity to perform all measurements of the
variables defined in the standard NBR IEC $60601-2-19$ and communicate them to a computer allowing the
transmission of measurement data and reception of commands for remote adjustment. The development of
this system creates a nationalized alternative with cheaper and more suitable features for the metrological
tests required. As a result, we obtained a micro-controlled portable and useful modular prototype, which
measures temperature, relative humidity, oxygen concentration, acoustic noise and air speed. The calibration
results show that the maximum relative error and measurement uncertainty for the subsystems of temperature,
relative humidity, acoustic noise and $O_{2}$ concentration were respectively $\left(-0.42 ; 0.05^{\circ} \mathrm{C}\right)$, $\left.\pm 0.72 ; 0.24 \%\right) R H$,
(-5.17; 0.68 dB) and $(1.28 ; 2.2 \%)$. These results indicate that the analyzer presents a metrological quality
compatible with the regulatory requirements established. Keywords: Evaluation of medical equipment, Metrology, Clinical engineering. 


\section{Extended Abstract}

\section{Introduction}

Neonatal incubators were created to increase the survival chances of premature newborns (NB). They basically regulate temperature, humidity and oxygen concentration, providing the ideal environmental conditions for the NB development. However, a malfunction may expose the NB to several sources of risk and may cause serious harm, even death.

To minimize such risk situations, the incubators should be kept in good condition through periodic inspections to verify the minimum requirements of performance and safety. To this end, many inspection tests involve the measurement of multiple physical magnitudes. Despite the benefits of automating the process of measuring, automatic instruments (also called analyzers) are usually imported and expensive. Furthermore, there are problems of usability and unsuitable features in some cases such as the use of a wire heated sensor in an oxygen-rich environment.

In order to overcome these problems, we have developed a low cost incubator analyzer with the capacity to perform all measurements of the variables defined in the standard NBR IEC 60601-2-19 and communicate them to a computer, allowing the transmission of measurement data and reception of commands for remote adjustment.

\section{Material and methods}

The prototype was developed in five phases: 1) design, 2) experimental assemblies and preliminary testing, 3) design and assembly of printed circuit boards $(P C B s), 4)$ final tests and 5) calibration.

In the design phase the metrological requirements of several measurement subsystems were collected according to standard NBR IEC 60601-2-19 (Associação..., 2000). These requirements are summarized in Table 1. The microcontroller architecture and the measurement subsystems were chosen to take into account the cost vs. benefit and appropriateness for the defined requirements. The experimental assemblies and preliminary tests have consolidated the design of the analyzer hardware and software, especially in the discrete circuitry such as in the A-weighted filter. The software was designed in a hierarchical way where basic functions such as programming/initialization, reading or writing in the devices were developed as firmware while the sampling, the interface, the data transfer and the remote mode were developed as functional modules at a higher layer.

For the sensing of temperature, NTC thermistors (Betatherm, model 10K3A1B) and a 24-bit resolution A/D converter were used. The air speed is captured by sensors (Omron, model D6FW01A1), with measurement range from zero to $1 \mathrm{~m} / \mathrm{s}$, uncertainty of $5 \%$ of full scale -FS, and analog output of 1 to $5 \mathrm{~V}$. In this case, the 12-bit A/D converter of the microcontroller was used.

Humidity sensing is carried out by the SHT75 provided by Sensirion with a digital serial output (14-bit internal $A / D$ converter) with measurement uncertainty of less than $\pm 4 \%$ for the range of 0 to $100 \%$. On the capture of acoustic noise, the A-weighted filter was assembled with RC discrete components and the RMS value was obtained by the AD637. In this case, the internal A/D converter of the microcontroller was also used. The oxygen concentration is measured by a paramagnetic sensor (Servomex, model Paracube). According to the manufacturer, its maximum measurement uncertainty is $0.5 \%$ for the range of 0 to $100 \%$, with a digital output compatible with standard UART. A portable prototype of practical use was assembled in phase three. The prototype was calibrated in specialized laboratories, following technical standards (Table 1, column 2) and with standard instruments calibrated and traced. Table 2 shows the technical data concerning the instruments used in calibrations.

The maximum figures for errors and measurement uncertainties found in the calibration process are presented in Table 3. For the subsystem of air velocity, comparative measurements showed a maximum error of $0.12 \mathrm{~m} / \mathrm{s}$ and maximum standard deviation of $0.012 \mathrm{~m} / \mathrm{s}$ for the sensor under test and $0.011 \mathrm{~m} / \mathrm{s}$ for the standard.

The interchangeability of the temperature and acoustic noise measurement subsystems were verified by Monte Carlo simulations. The response equations of the subsystems were used to verify the propagation of uncertainties due to circuit components tolerances. Variations in the response of the AD637 are made based on information from their datasheet.

\section{Results}

The functional block diagram of the system is shown in Figure 1. The analyzer architecture and its external interfaces can be seen. The physical aspect of the prototype is shown in Figure 2. The sensors were mounted on sliding blocks and rods to facilitate their placement within the incubator hood. To illustrate the use of the analyzer, Figure 3 presents measurements inside neonatal incubators and Figure 4 shows compliance report fragments issued by the automation software. The results of the uncertainties analysis for interchangeability verification of the temperature and acoustic noise subsystems are presented in Tables 4 and 5.

\section{Discussion and conclusion}

The maximum relative errors found in the various subsystems of measurement (Table 3), and more significantly in the sound noise and oxygen concentration, point to the need for corrections. In this respect, the micro-controlled structure facilitates the programming of correction functions that can be modeled from the calibration points.

The measurement uncertainties estimated in the calibration process (Table 3) in general corroborate the metrological quality required for the prototype (Table 1). The calibration of the oxygen sensor is an exception due to the inadequacy of the standard instrument which has an uncertainty of $1.6 \%$, exceeding the limit laid down at $0.5 \%$. The same was also observed for the air speed subsystem.

The analysis of measurements in the incubators identified various non-conformities. Measurements of relative humidity and oxygen concentration were the most discrepant with maximum errors of $7.1 \%$ UR and $18.4 \% \mathrm{O}_{2}$ respectively. This reinforces the need for such evaluation, facilitating the identification of important sources of risk to patients and users.

According to Table 4, the interchangeability of the temperature measurement subsystem $\left(U \leq 0.05^{\circ} \mathrm{C}\right)$ was only obtained with the reduced tolerance of fixed resistors and thermistors to $0.02 \%$ and $0.3 \%$.

As a microphone with sufficiently low tolerance was not found, this was the only device of the acoustic noise measurement subsystem that did not allow interchangeability. An adjustable gain amplifier was used to minimize possible deviations. The resulting uncertainty, as shown by $D$ in Table 5 , depends on the uncertainty of the instruments used in this setting ( $V_{0}$ and NS). The maximum deviation, resulting from variations in the components of the A-weighted filter occurred at $10 \mathrm{~Hz}\left(D_{2(10 \mathrm{~Hz}}\right)$. The deviation due to the converter AD637 $\left(D_{3}\right)$ was estimated for the circuit with and without external offset and scale adjustment. The overall confidence interval was estimated by linear and quadratic sum for the different scenarios (frequency, adjustments of the microphone and AD637). All confidence intervals estimated for this subsystem met the tolerance limits prescribed by standard IEC 61672-1. 


\section{Introdução}

O nascimento prematuro se constitui em grave problema de saúde pública e um importante fator para o aumento da mortalidade e deficiências em neonatos (Bercini, 1994). Geralmente os nascidos com menos de 37 semanas de gestação têm baixo peso, pigmentação incompleta, dificuldades em regular a temperatura corporal e absorver o oxigênio da atmosfera (Rego e Anchieta, 2005). Se a temperatura e o nível de oxigênio não forem regulados artificialmente, o recém-nascido $(\mathrm{RN})$ passa a correr risco de morte ou de sofrer sequelas neurológicas irreversíveis.

Para garantir maiores chances de sobrevivência dos RN prematuros, foram criadas em meados do século XIX as incubadoras neonatais, que proporcionam as condições ambientais próximas ao ideal para o desenvolvimento dos mesmos (Rodrigues e Oliveira, 2004). Tais equipamentos regulam a temperatura, a umidade relativa e a concentração de oxigênio nos níveis estabelecidos por uma equipe médica, embora outras variáveis como fluxo de ar e ruído também necessitem de avaliação. Entretanto, se os sistemas de controle não forem capazes de manter estáveis tais grandezas, o RN estará sujeito a diversas fontes de risco. Mudanças na temperatura prescrita podem provocar hipotermia, hipertermia e apneia. Variações do fluxo de ar podem aumentar a troca de calor entre o $\mathrm{RN}$ e o ambiente (Kim et al., 2002). Baixos níveis de oxigênio estão relacionados a danos cerebrais e morte, enquanto que altos níveis podem causar fibroplasia retrolental (Ashton et al., 1953). Há, ainda, os riscos devido ao ruído produzido pelo equipamento ou no ambiente da UTI neonatal. O RN pode apresentar perda auditiva ou surdez caso o nível de ruído seja elevado e o tempo de exposição prolongado (Carvalho e Pereira, 1998; Falk e Farmer, 1973).

Inspeções periódicas, baseadas em normas, estabelecem os requisitos mínimos de desempenho e segurança e envolvem a medição de múltiplas grandezas em condições predeterminadas. A execução dos ensaios para avaliação de incubadoras neonatais, previstos na norma NBR IEC 60601-2-19 (Associação..., 2000), requer um termômetro digital com cinco canais, um higrômetro, um decibelímetro, um anemômetro e um analisador de oxigênio. Além disso, necessita de mão de obra especializada e um longo tempo para a execução e análise dos dados. A utilização de um método automático para a medição destas grandezas trará como vantagens: praticidade, portabilidade, facilidade e confiabilidade na medição, além da redução de custos e de tempo.

Equipamentos projetados para efetuar medições de múltiplas variáveis, com a finalidade de avaliar um tipo específico de EM são denominados analisadores. No caso das incubadoras, os analisadores disponíveis no mercado são importados, com custo superior a R\$ 45 mil.

Além disso, um dos analisadores de incubadoras mais utilizados no país (Fluke Biomedical, modelo $\mathrm{INCU}^{\mathrm{TM}}$ ) apresenta inconvenientes de usabilidade e características construtivas inadequadas. O modelo traz os sensores encaixados em pontos fixos do próprio equipamento e em número insuficiente. Isso dificulta a distribuição dos sensores no ambiente interno da incubadora, obriga o reposicionamento dos mesmos e a repetição dos ensaios para que seja atingida a quantidade de pontos de medição estabelecidos na norma. Como resultado dessas inadequações, o tempo total de execução de um ensaio pode chegar a 15 horas, considerando o período para estabilizar a temperatura, o número de repetições dos ensaios e as medições previstas. Ainda no que diz respeito à medição de temperatura, esse analisador apresenta especificações de incertezas das medições de $0,5^{\circ} \mathrm{C}$, contrariando o limite de $0,05^{\circ} \mathrm{C}$ prescrito na norma brasileira.

Outro inconveniente que esse instrumento apresenta é a utilização de sensor de fio aquecido para medição de fluxo de ar, diretamente exposto ao microclima da incubadora. Tal fato pode ocasionar ignição devido ao enriquecimento com oxigênio. Além disso, o uso de um único sensor com posicionamento fixo dificulta a comprovação de uma distribuição de fluxo homogênea.

Assim, visando superar os inconvenientes construtivos e de usabilidade, foi desenvolvido um analisador de incubadoras com capacidade de efetuar simultaneamente todas as medições das variáveis definidas na norma e com possibilidade de comunicação com um computador. Esta última funcionalidade permite a transmissão dos dados medidos e a recepção dos comandos para ajustes remotos no analisador.

\section{Materiais e Métodos}

O protótipo foi desenvolvido em cinco fases: 1) concepção, 2) montagens experimentais e testes preliminares, 3) projeto e montagem em placas de circuitos impressos, 4) testes finais e 5) calibração.

$\mathrm{Na}$ fase de concepção registraram-se os requisitos metrológicos dos diversos subsistemas de medição, tais como número de canais, faixa e incerteza de medição, de acordo com a norma NBR IEC 60601-2-19. Os subsistemas de medição de temperatura, velocidade do ar, umidade relativa, concentração de oxigênio e nível de ruído foram projetados em conformidade com os requisitos registrados, mas também avaliando custos, disponibilidade e complexidade. Ainda nesta 
fase foram esboçadas diversas opções de alojamento dos circuitos e da estrutura de posicionamento dos sensores, visando o uso prático do protótipo.

Para tornar a execução dos ensaios prescritos na norma mais eficiente, os subsistemas de temperatura e velocidade do ar foram planejados com múltiplos canais, possibilitando medições simultâneas em diferentes pontos no interior da cúpula da incubadora. Os demais subsistemas foram concebidos com um único canal.

Outra opção do projeto foi o uso de um microcontrolador para controle e inicialização dos subsistemas, aquisição dos dados de medição, interface com o usuário por meio de display e teclas, e comunicação com um computador, podendo funcionar isoladamente ou em modo remoto. A escolha do microcontrolador foi baseada no número de portas necessárias para interligação com os subsistemas, capacidade de memória compatível com o tamanho do firmware do analisador, presença de um conversor $\mathrm{AD}$ com resolução suficiente para atender aos subsistemas com saída analógica, entre outras.

No subsistema de temperatura, termistores NTC (Betatherm, modelo 10K3A1B) foram escolhidos em função da relação custo/benefício em comparação com outros tipos de sensores. Um circuito de condicionamento foi projetado para alimentar os termistores, linearizar as suas curvas e evitar ruídos de alta frequência.

Como a alimentação impacta diretamente na qualidade dos sinais de medição, foi necessária a inclusão de um circuito de precisão para estabilizar a tensão. A etapa de linearização foi obtida com a associação de um resistor fixo $R_{1}$ em série com o termistor $R(T)$. A tensão de saída, função da temperatura $T$, foi tomada sobre $R_{1}$. A Equação 1 expressa essa tensão quando normalizada pela tensão de alimentação $V_{\text {Ref }}$ e a Equação 2 enfatiza sua forma linear, onde $\varepsilon(T)$ é o erro entre os valores real e o aproximado.

Desta forma, o problema da linearização se resumiu à definição de um resistor $R_{1}$ que minimizasse $\varepsilon(T)$ ao longo da faixa de medição. Em termos práticos, para encontrar-se a solução expressa na Equação 3, desprezou-se $\varepsilon(T)$, tomou-se três pontos distintos de temperatura, que foram escolhidos estrategicamente como os extremos e o ponto médio da faixa de medição ( $T_{\mathrm{L}}, T_{\mathrm{H}}$ e $T_{\mathrm{M}}$, respectivamente) e resolveu-se o sistema ( $a, b$ e $R_{1}$ como incógnitas). Considerou-se ainda, as condições de contorno relativas à faixa de tensão a ser disponibilizada para o ADC e a máxima corrente que permitia a operação dos termistores sem o efeito de autoaquecimento.

Um filtro passa baixas (PB) foi projetado para funcionar em cascata com a etapa de linearização para evitar possíveis ruídos de alta frequência. Devido às elevadas constantes de tempo, característica dos sistemas de medição de temperatura em meios gasosos, a frequência de corte do filtro foi fixada em torno de $16 \mathrm{~Hz}$. Nesse circuito de condicionamento foram utilizados resistores de $1 \%$ de precisão e de $50 \mathrm{ppm} /{ }^{\circ} \mathrm{C}$ de estabilidade térmica.

$\frac{v_{0}(T)}{V_{\text {Ref }}}=\frac{1}{1+\frac{R(T)}{R_{1}}}$

$\frac{v_{\mathrm{o}}(T)}{V_{\mathrm{Ref}}}=a T+b-\varepsilon(T)$

$R_{1}=\frac{R\left(T_{L}\right) R\left(T_{M}\right)+R\left(T_{M}\right) R\left(T_{H}\right)-2 R\left(T_{L}\right) R\left(T_{H}\right)}{R\left(T_{L}\right)+R\left(T_{H}\right)-2 R\left(T_{M}\right)}$

Embora a resolução de 12 bits do conversor interno do microcontrolador fosse adequada, os seus oito canais eram insuficientes para o total de 10 variáveis analógicas ( 5 de temperatura, 4 de velocidade do ar e 1 de nível de ruído). Assim, pela disponibilidade e baixo custo dos modernos conversores $\mathrm{AD}$ e ainda, pela perspectiva de desenvolvimento de outros instrumentos de maior precisão, optou-se por utilizar um conversor AD Sigma-Delta de 24 bits.

$\mathrm{Na}$ fase dos testes finais foram identificados erros significativos na leitura das temperaturas, introduzidos pela técnica de linearização. A solução adotada foi utilizar o valor de $v_{\mathrm{o}}$, obtido do conversor $\mathrm{AD}$, para calcular, por software, o valor de $R(T)$ e aplicá-lo na equação de Steinhart e Hart (1968), para determinar $T$, conforme a Equação 4.

$$
\begin{gathered}
T=\frac{1}{a+b \ln R(T)+c \ln ^{3} R(T)} \\
R(T)=\left(\frac{V_{\mathrm{Ref}}}{v_{\mathrm{o}}}-1\right) R_{\mathrm{lVN}}
\end{gathered}
$$

onde:

- $a, b$ e $c$ são constantes especificadas pelo fabricante;

- $R_{1 \mathrm{VN}}$ é o valor nominal de $R_{1}$

Foi realizada uma análise, por Simulação de Monte Carlo (SMC), para verificar a permutabilidade do subsistema, isto é, a possibilidade de substituir seus componentes sem a necessidade de ajustes ou calibrações, por exemplo, em um reparo ou na montagem de novas unidades. A resposta de todo o circuito de condicionamento foi usada para simular $v_{0}$ em função dos valores dos componentes e da frequência do sinal de temperatura (Equação 5). Cada componente foi simulado com distribuição normal, com média igual ao valor nominal e desvio padrão (DP) proporcional à tolerância. A Equação 4 foi utilizada 
para encontrar a variável de medição $T$ com média e DP resultantes. Testes de hipótese - Kolmogorov, Jarque-Bera e Lilliefors - foram realizados para comprovar a normalidade da distribuição resultante. A incerteza expandida foi estimada a partir da SMC. As simulações foram executadas para duas décadas acima e abaixo da frequência de oscilação da temperatura da incubadora sob teste. Os valores nominais do termistor a $20{ }^{\circ} \mathrm{C}$ e $40{ }^{\circ} \mathrm{C}$ foram obtidos por tabela fornecida pelo fabricante.

$$
\left|\frac{v_{0}}{V_{\text {Ref }}}\right|=\frac{\frac{R_{1 R_{\text {in }}}}{\left(R_{1}+R(T)\right)\left(R_{1} / / R(T)+R_{2}+R_{i n}\right)}}{\sqrt{\left(2 \pi f\left(\left(R_{1} / / R(T)+R_{2}\right) / / R_{\text {in }}\right) C\right)^{2}+1}}
$$

onde:

- $R_{\text {in }}$ é a resistência de entrada do ADC;

- $R_{2}$ e $C$ são os componentes do filtro PB.

Para comprovar a distribuição normal e determinar a constante de proporcionalidade entre a tolerância e o DP dos componentes, foi executado um experimento. Foram medidos 55 resistores de $1.000 \Omega$ e $5 \%$ de tolerância e o resultado foi uma distribuição normal com média $995 \Omega$ e DP de 7,33 $\Omega$. Considerando o DP da média, a incerteza do medidor e o desvio da média em relação ao valor nominal, a incerteza padrão combinada para esse experimento foi estimada em cerca de $5 \Omega$, levando a um fator de 10 , quando comparada com a tolerância de $5 \%(50 \Omega)$. Um fator mais conservador foi encontrado utilizando-se a Equação 6 (Howe, 1969), que resultou em $k=4,37$. Por falta de informação sobre o nível de confiança na fabricação dos termistores, dos resistores e do capacitor, foi adotado $k=4$ para as simulações do subsistema de temperatura.

$k=\sqrt{\frac{(N-1)(1+1 / N) Z_{(1-p) / 2}^{2}}{\chi_{\gamma, N-1}^{2}}} \cong 4,37$

para:

- $N=55$ (número de amostras);

- $p=99,8 \%$ (proporção da população dentro da tolerância);

- $\gamma=99,9 \%$ (nível de confiança);

- $\mathrm{Z}_{(1-p) / 2}^{2}=3,09$ (valor crítico da função densidade de probabilidade (PDF) normal que excede $(1-p) / 2$;

- $\chi_{\gamma, N-1}^{2}=27,47$ (valor crítico da PDF quiquadrado, com $(N-1)$ graus de liberdade, que excede a probabilidade $\gamma$.

No subsistema de velocidade do ar, evitou-se a utilização de técnicas de anemometria por dispersão de calor direta, isto é, o uso de fio aquecido, devido ao risco de incêndio em ambientes ricos em oxigênio.
Escolheu-se um sensor montado no próprio circuito integrado (Omron DF6W01A). Esse tipo de tecnologia é conhecido como MEMS - MicroElectroMechanical Systems (Maluf e Williams, 2004). A opção simplificou o projeto, pois integrou o sensoriamento e o condicionamento do sinal em um único componente. Como os ensaios relativos a essa grandeza estão associados a um limiar de conformidade normativa de $0,35 \mathrm{~m} / \mathrm{s}$ (NBR IEC 60601-2-19), foi escolhido um sensor com faixa de zero a $1 \mathrm{~m} / \mathrm{s}$ e para a conversão $\mathrm{AD}$ foi utilizado o ADC de 12 bits do próprio microcontrolador. Embora a norma seja omissa na especificação da incerteza deste subsistema, foi adotada uma incerteza máxima de $\pm 0,05 \mathrm{~m} / \mathrm{s}$.

A mesma estratégia de integração foi adotada para o subsistema de medição de umidade relativa (UR). Neste caso buscou-se um sensor com a maior faixa de medição possível e com uma incerteza máxima de $\pm 5 \%$ UR. Outra vez, a norma é omissa na caracterização metrológica do medidor. O sensor escolhido (Sensirion SHT75) possui 2 pinos de alimentação, 2 pinos de comunicação, ADC interno de 14 bits e incerteza máxima de $\pm 4 \%$ UR na faixa de zero a $100 \%$ UR.

Para a medição do nível de ruído, optou-se pela transdução através de um microfone de eletreto, por apresentar a resposta em frequência mais plana na faixa de utilização $(20 \mathrm{~Hz}$ a $20 \mathrm{kHz})$. O microfone escolhido (Panasonic WM-61A), além de baixo custo, possui sensibilidade de 19,7 $\pm 8,5 \mathrm{mV} / \mathrm{Pa}$ e relação sinal/ruído $\geq 62 \mathrm{~dB}$. Em seguida foi necessário condicionar o sinal através de um filtro ponderado tipo A. Este filtro possui uma resposta em frequência semelhante à do ouvido humano e, de acordo com a norma IEC 61672-1 (International..., 2002), pode ser implementado a partir da Equação 7. Desta forma, o filtro foi construído a partir de quatro filtros elementares ligados em cascata, um PB de segunda ordem com frequência de corte $f_{\mathrm{c}}=f_{1}$, um passa altas (PA) de segunda ordem $\operatorname{com} f_{\mathrm{c}}=f_{4}$ e mais dois filtros PA de primeira ordem $\operatorname{com} f_{\mathrm{c}}=281,84 \mathrm{~Hz}$. A interação entre os dois polos dos dois últimos filtros resulta nas frequências $f_{2}$ e $f_{3}$. Um amplificador de ganho ajustável foi usado para se obter o ganho de zero dB em $1 \mathrm{kHz}\left(A_{1000}\right)$ e compensar o desvio na sensibilidade do microfone em relação ao valor nominal de $19,7 \mathrm{mV} / \mathrm{Pa}$. Por fim, o valor eficaz do sinal, obtido utilizando um conversor AD637, foi digitalizado pelo ADC interno do microcontrolador e convertido em dB por software.

$$
\begin{aligned}
A(f)= & 20 \log \left[\frac{f_{4}^{2} f^{4}}{\left(f^{2}+f_{1}^{2}\right)\left(f^{2}+f_{2}^{2}\right)^{1 / 2}\left(f^{2}+f_{3}^{2}\right)^{1 / 2}\left(f^{2}+f_{4}^{2}\right)}\right] \\
& -A_{1000}
\end{aligned}
$$


$\operatorname{com} f_{1}=20,60 \mathrm{~Hz}, f_{2}=107,7 \mathrm{~Hz}, f_{3}=737,9 \mathrm{~Hz}$, $f_{4}=12.194 \mathrm{~Hz}$ e $A_{1000}=-2,000 \mathrm{~dB}$.

A faixa de medição foi estabelecida entre 45 a $90 \mathrm{~dB}$ para atender aos valores limites estabelecidos na norma. A incerteza máxima pretendida de 1,4 dB(A), para a frequência de $1 \mathrm{kHz}$, segue a prescrição da norma IEC 61672-1 (International..., 2002). Durante o processo de calibração verificou-se a resposta em frequência entre $20 \mathrm{~Hz}$ e $20 \mathrm{kHz}$ e a indicação do nível sonoro na faixa de medição definida. Observou-se ainda, se tais respostas atendiam aos limites de tolerância tabelados na seção 5.4 .2 da norma IEC 61672-1.

A permutabilidade deste subsistema também foi verificada, porém, admitiu-se o ajuste de compensação devido à grande margem de variação da sensibilidade do microfone. Por simplificação, o subsistema foi dividido em três blocos: 1) microfone e amplificador de ganho ajustável; 2) filtro ponderado tipo A; e 3) conversor AD637.

A incerteza do primeiro bloco foi estimada através de SMC, considerando-se o desvio entre as sensibilidades experimental e projetada, conforme a Equação 8, onde $V_{0}$ é tensão na saída do amplificador e $N S$ é o nível de pressão sonora no microfone. A média e o DP, associados à Função Densidade de Probabilidade (PDF) normal destas variáveis, foram obtidos a partir dos valores e das incertezas dos instrumentos utilizados no procedimento de ajuste do ganho do amplificador.

$$
D_{1}=20 \log \frac{S_{E x p}}{S_{\text {Pro }}}, S_{E x p}=\frac{V_{\mathrm{o}}}{P_{\mathrm{o}} 10^{N S / 20}}
$$

com:

- $S_{\mathrm{Pr} o} \cong 5,97 \mathrm{~V} / \mathrm{Pa}$;

- $P_{\mathrm{o}}=2 \cdot 10^{-5} \mathrm{~Pa}$

A estimativa da incerteza do filtro tipo A também foi realizada por SMC, considerando-se o desvio entre o ganho ideal (Equação 7) e o ganho experimental, calculado analiticamente, através de sua função de transferência, conforme Equação 9, onde $Z_{\mathrm{n}, \mathrm{m}}$ é a impedância vista do $n$-ésimo ponto do $m$-ésimo filtro de segunda ordem, com os dois filtros de primeira ordem considerados como um único, de segunda ordem. A expressão do ganho experimental $\left(A_{\text {Exp }}\right)$ foi validada por comparação com valores obtidos no simulador Electronics Workbench. Por falta de informação sobre o nível de confiança na fabricação de resistores e capacitores, foi utilizado o fator $k=4,37$ entre a tolerância e o respectivo DP, conforme Equação 6. A simulação foi executada para a faixa de $10 \mathrm{~Hz}$ a $20 \mathrm{kHz}$, cobrindo todas as frequências tabeladas na seção 5.4.2 da norma IEC 61672-1.

$$
\begin{aligned}
& D_{2}=A_{\text {Exp }}(f)-A(f) \\
& A_{E x p}(f)=20 \log \left[\prod_{\substack{n=2,4 \\
m=1,2,3}}\left|\frac{Z_{n, m}}{Z_{n-1, m}}\right|\right]-A_{1000}
\end{aligned}
$$

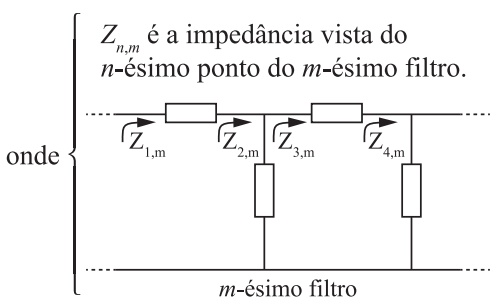

A incerteza $\left(D_{3}\right)$ do conversor AD637 foi estimada com base nas informações do datasheet do fabricante. Foram encontradas duas componentes: a incerteza devido aos erros de zero (offset) e de escala, e a incerteza devido ao erro de conversão. Na primeira componente, a incerteza é $0,5 \mathrm{mV}+0,2 \%$ da leitura, para o circuito sem ajustes externos de zero e de escala, ou $0,25 \mathrm{mV}+0,05 \%$ da leitura, para o circuito com ajustes externos. A outra componente é fornecida em valor percentual da leitura, por meio de um gráfico, em função da frequência do sinal e da capacitância do capacitor de integração, incluindo as variações relativas às tolerâncias dos componentes. O capacitor de integração foi calculado em função do tempo de resposta da conversão, especificado em $1 \mathrm{~s}$ (um segundo). As parcelas foram calculadas para o pior caso, isto é, tensão e frequência do sinal de entrada mínimas, e capacitor de integração de $8 \mu \mathrm{F}$. As parcelas foram somadas linearmente, em módulo, por desconhecimento dos seus fatores de abrangência.

A incerteza expandida resultante foi calculada de duas formas. No pior caso (soma linear), desconhecendo-se o fator de abrangência de $D_{3}$, a incerteza expandida dos dois primeiros blocos foi estimada, com $95 \%$ de confiança $(k=2)$, e então, somada em modulo com $D_{3}$. Na outra forma (soma quadrática), admitiu-se o fator de abrangência de $D_{3}$ como $k=2$, e assim, a incerteza expandida resultante foi estimada através da incerteza padrão combinada dois primeiros blocos e a incerteza padrão do terceiro, para o mesmo nível de confiança de $95 \%(k=2)$.

Para o monitoramento de oxigênio, embora a norma NBR IEC 60601-2-19 faça referência à ISO 7767 (International..., 1997), a norma atualmente em vigor é a ISO 21647 (International..., 2004). Esta última prescreve uma incerteza de $2,5 \%+2,5 \%$ da leitura, em fração de volume, para os monitores de oxigênio destinados ao uso em humanos, e ainda fixa a incerteza dos padrões de calibração em $1 / 5$ destes valores. Desta forma, foi estabelecida a incerteza máxima pretendida para o subsistema de medição da concentração de oxigênio em $0,5 \%$, na faixa de $0 \%$ a $100 \%$. 
Para a medição da concentração de oxigênio utilizou-se uma célula permanente que funciona através da propriedade paramagnética do oxigênio, eliminando-se o uso de células eletroquímicas que necessitam de troca periódica devido ao esgotamento do reagente.

O sensor adotado foi o Paracube da Servomex, que apresenta saída digital com incerteza máxima de $0,5 \% \mathrm{O}_{2}$. Neste caso, a integração do sensor com os circuitos eletrônicos também foi perseguida como meio de simplificação do sistema. Os requisitos utilizados para os subsistemas de medição estão sumarizados na Tabela 1 .

As fases de montagens experimentais e testes preliminares ajudaram na consolidação da concepção do hardware e do software do analisador, especialmente dos circuitos discretos, como no filtro com ponderação tipo A. O software foi projetado de forma hierarquizada, onde funções básicas como programação, inicialização, leitura ou escrita de dispositivos, foram desenvolvidas como um firmware enquanto os módulos funcionais de amostragem, interface, transferência de dados e modo remoto, como uma camada superior.

O módulo de amostragem se ocupa das leituras dos subsistemas de medições, processa os dados brutos, fazendo correções, e os convertendo para as unidades apropriadas. O módulo de interface é responsável pela leitura do teclado de três teclas e escrita do display LCD, bem como pela navegação nos itens de um menu. O módulo de transferência envia o conteúdo da memória EEPROM para um computador, após um período de funcionamento onde os dados de medições são acumulados nesta memória.

O módulo remoto adiciona uma funcionalidade especial ao analisador: receber comandos e enviar dados de medição, em tempo real, para um computador. Com essa funcionalidade é possível ampliar a capacidade de exibição das medições (tabelas, gráficos etc.) e de automatizar ensaios, através da execução de um aplicativo apropriado, no computador. Isto permite ao sistema desenvolvido automatizar as avaliações metrológicas de equipamentos médicos.
O sistema gerencia um número configurável de conexões que permite a aquisição de dados de diversos instrumentos ou equipamentos. Possui uma interface óptica de reconhecimento de dígitos, que permite a leitura dos valores apresentados no display do equipamento ou instrumento que não possui outro canal de comunicação. As funções específicas de monitoramento e de avaliação são organizadas em módulos que podem ser adicionadas dinamicamente ao sistema, de acordo com sua configuração. Também é possível configurar instruções detalhadas de ajustes operacionais do EM sob teste, que são apresentadas ao usuário antes de cada ensaio. Um relatório de conformidade técnica é emitido no final da avaliação.

Quase todos os subsistemas foram calibrados em laboratórios confiáveis, seguindo normas técnicas e com padrões calibrados e rastreados. A única exceção foi o subsistema de velocidade do ar que apresentou problemas técnicos na tentativa de calibração. Dessa forma, foi realizada apenas uma comparação com equipamento comercial calibrado, mas sem as condições ideais. Na Tabela 2 são apresentados dados técnicos referentes aos padrões utilizados nas calibrações.

Embora os resultados das calibrações fossem o meio mais seguro de comprovação da qualidade das medições, foram realizadas medições do microclima de algumas incubadoras para exemplificar a utilização do protótipo. Neste caso, os ensaios seguiram as prescrições da norma NBR IEC 60601-2-19 e as instruções operacionais dos fabricantes das incubadoras.

\section{Resultados}

O diagrama em blocos funcionais do sistema é apresentado na Figura 1. O aspecto físico do protótipo é apresentado na Figura 2. A estrutura de posicionamento dos sensores é constituída de hastes e blocos de madeira. As hastes laterais são deslizantes e permitem o movimento vertical (1). Um bloco é fixado no centro da estrutura (2) e os outros (3) são distribuídos sobre as hastes laterais. Os blocos periféricos podem deslizar no sentido horizontal. Guias tubulares de

Tabela 1. Especificações utilizadas para os subsistemas de medição. Table 1. Specifications used for the measurement subsystems.

\begin{tabular}{|c|c|c|c|c|c|c|}
\hline Subsistema & Referência normativa & Tecnologia & Canais & Faixa & $\begin{array}{c}\text { Incerteza } \\
\text { expandida }\end{array}$ & Unidade \\
\hline Temperatura & NBR IEC 60601-2-19 & NTC & 5 & 20,00 a 40,00 & $\pm 0,05$ & ${ }^{\circ} \mathrm{C}$ \\
\hline Velocidade do ar & NBR IEC 60601-2-19 & MEMS & 4 & 0,00 a 1,00 & $\pm 0,05$ & $\mathrm{~m} / \mathrm{s}$ \\
\hline Umidade relativa & $\begin{array}{l}\text { Não citada. Foi adotado um } \\
\text { valor típico }\end{array}$ & MEMS & 1 & 0 a 100 & \pm 5 & $\%$ UR \\
\hline Nível de ruído & IEC 61672-1 & Circuito discreto & 1 & 45,0 a 90,0 & $\pm 1,4$ & $\mathrm{~dB}(\mathrm{~A})$ \\
\hline Concentração de $\mathrm{O}_{2}$ & ISO 21647 & MEMS & 1 & 0,0 a 100,0 & $\pm 0,5$ & $\%$ \\
\hline
\end{tabular}


Tabela 2. Instrumentação utilizada nas calibrações/comparações.

Table 2. Instrumentation used in calibrations/comparisons.

\begin{tabular}{|c|c|c|}
\hline Subsistema & Instrumento padrão & Outros instrumentos \\
\hline Temperatura & $\begin{array}{l}\text { Termômetro digital com termistor } \\
\text { Fabricante: Hart Scientific } \\
\text { Modelos: } 1504 \text { e } 5642-\mathrm{X} \\
\text { Incerteza: } 0,001^{\circ} \mathrm{C} \\
\text { Rastreabilidade: NVLAB }\end{array}$ & $\begin{array}{c}\text { Banho térmico } \\
\text { Fabricante: Nova Ética } \\
\text { Modelo: } 521 / 2 \mathrm{DCE} \\
\text { Estabilidade térmica: } 0,03^{\circ} \mathrm{C}\end{array}$ \\
\hline Umidade relativa & $\begin{array}{l}\text { Termohigrômetro digital } \\
\text { Fabricante: Testo } \\
\text { Modelo: } 608 \mathrm{H} 1 \\
\text { Incerteza: } 0,2 \% \text { UR }(k=2) \\
\text { Rastreabilidade: RBC - PUCRS T 0652/2009 }\end{array}$ & $\begin{array}{l}\text { Câmara de climatização } \\
\text { Fabricante: Nova ética }\end{array}$ \\
\hline Nível de ruído & $\begin{array}{l}\text { Medidor de nível sonoro } \\
\text { Fabricante: Minipa } \\
\text { Modelo: MSL } 1352 \mathrm{~A} \\
\text { Incerteza: } 0,2 \mathrm{~dB}(\mathrm{~K}=2,07) \\
\text { Rastreabilidade: } \mathrm{RBC} \text { - CHOMPACK } 23584\end{array}$ & $\begin{array}{c}\text { Modelo: } 430 \mathrm{RD} \\
\text { Estabilidade: } \pm 1{ }^{\circ} \mathrm{C} \text { e } \pm 2 \% \text { UR. }\end{array}$ \\
\hline Concentração de oxigênio & $\begin{array}{l}\text { Medidor de concentração de oxigênio } \\
\text { Fabricante: Teledne } \\
\text { Modelo: TED } 60 \mathrm{~T} \\
\text { Incerteza: } 1,6 \%\end{array}$ & $\begin{array}{l}\text { Misturador (Blender) } \\
\text { Fabricante: Intermed } \\
\text { Modelo: Air O2 Blender }\end{array}$ \\
\hline Velocidade do ar & $\begin{array}{l}\text { Anemômetro digital } \\
\text { Fabricante: Testo } \\
\text { Modelo: } 425 \\
\text { Incerteza: } 0,08 \mathrm{~m} / \mathrm{s} \\
\text { Rastreabilidade: calibração de fábrica }\end{array}$ & \\
\hline
\end{tabular}

As incertezas expandidas estimadas correspondem a um intervalo de confiança de $95 \%$.

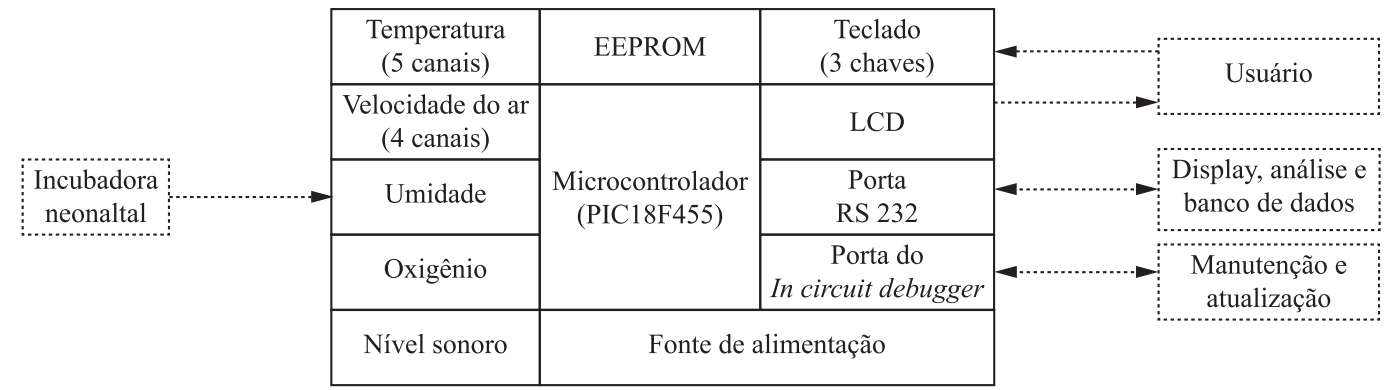

Figura 1. Sistema analisador de incubadoras.

Figure 1. Neonatal incubator analyzer system.

alumínio auxiliam no movimento das partes móveis. Um sensor de fluxo de ar e outro de temperatura são fixados em cada bloco periférico. Os demais sensores são fixados no bloco central. O circuito principal, o display, o teclado e as conexões foram alojados em uma caixa plástica de $110 \times 190 \times 65 \mathrm{~mm}$. O custo de produção do protótipo foi inferior a $10 \%$ do valor de um analisador importado.

Os resultados das calibrações são apresentados na Tabela 3. Devido à grande quantidade de dados, foram selecionados apenas os valores máximos. No caso do subsistema de velocidade do ar, não se pode definir a incerteza ou o fator $k$, entretanto, medidas comparativas apontaram um erro máximo de $0,12 \mathrm{~m} / \mathrm{s}$ e desvio padrão máximo de $0,012 \mathrm{~m} / \mathrm{s}$ para o sensor em teste e $0,011 \mathrm{~m} / \mathrm{s}$ para o instrumento padrão.

Algumas das medições realizadas no interior das incubadoras são apresentadas na Figura 3. As medições foram realizadas no Laboratório de Engenharia Clínica do NTS/IFBA em uma incubadora especialmente adquirida para o desenvolvimento do protótipo, com exceção da concentração de oxigênio, que foi realizada em uma incubadora externa, devido ao esgotamento da célula de oxigênio da primeira. A Figura 4 apresenta apenas partes do relatório de conformidade técnica em virtude de sua grande extensão. 


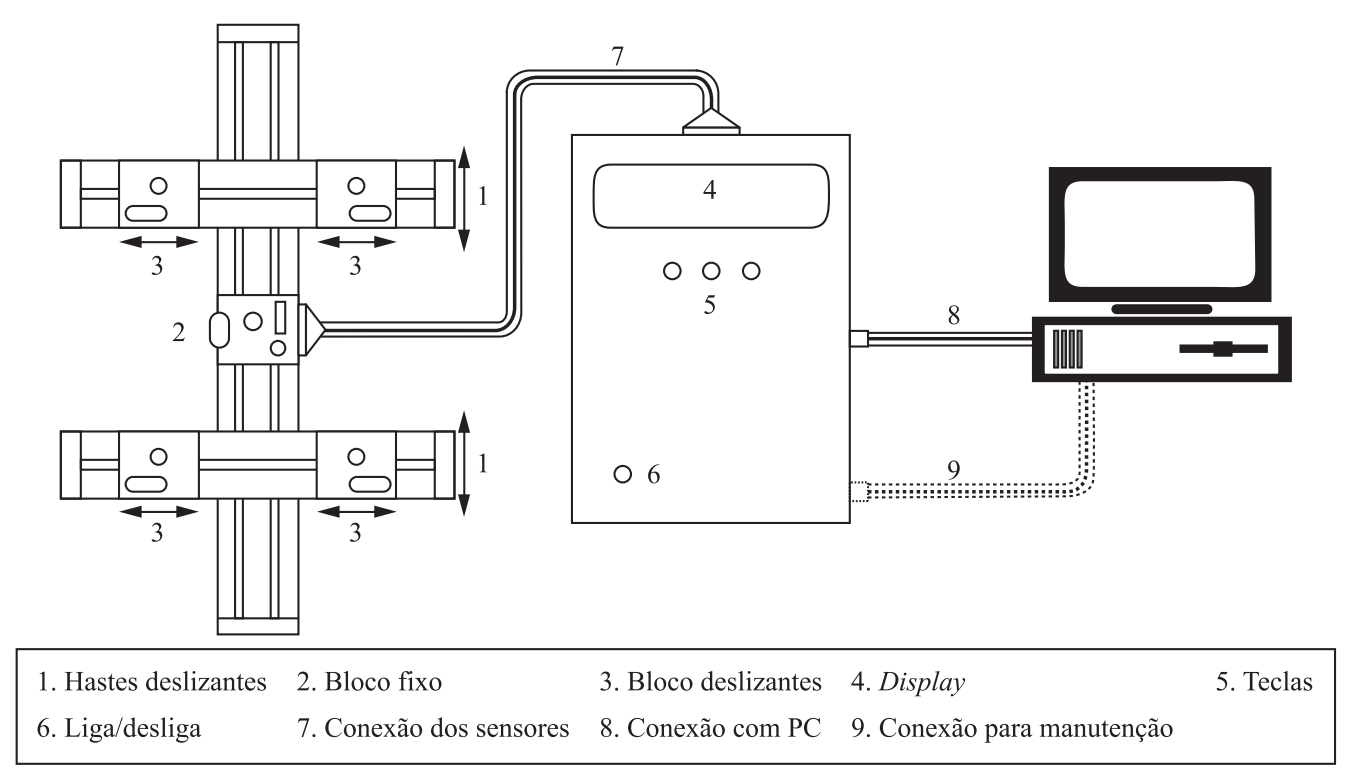

Figura 2. Disposição física do protótipo e detalhes da estrutura de posicionamento dos sensores.

Figure 2. Prototype physical layout and details of the sensors positioning structure.

Tabela 3. Resultados das calibrações.

Table 3. Calibration results.

\begin{tabular}{lcccc}
\hline \multirow{2}{*}{ Subsistema } & \multirow{2}{*}{ Erro relativo máximo (\%) } & \multicolumn{2}{c}{ Incerteza máxima (95\% de confiança) } \\
\cline { 3 - 5 } & & Fator $\boldsymbol{k}$ & Incerteza expandida $-\mathbf{U}$ & Unidade \\
\hline Temperatura (Ch1) & 0,36 & 2,00 & 0,05 & 0,05 \\
Temperatura (Ch2) & $-0,16$ & 2,00 & 0,05 & ${ }^{\circ} \mathrm{C}$ \\
Temperatura (Ch3) & $-0,42$ & 2,00 & 0,05 & $\%$ UR \\
Temperatura (Ch4) & 0,40 & 2,00 & 0,05 & dB \\
Temperatura (Ch5) & 0,30 & 2,00 & 0,24 & $\%$ \\
Umidade relativa & $\pm 0,72$ & 2,04 & 0,68 & 2,20 \\
Nível de ruído & $-5,17$ & 4,30 & & $\%$ \\
Concentração de $\mathrm{O}_{2}$ & 1,28 & 2,78 & & \\
\hline
\end{tabular}

'Percentagem sobre a média dos valores lidos nos padrões de referência

Os resultados das análises de incerteza para verificação da permutabilidade dos subsistemas de medição da temperatura e nível de ruído são apresentados nas Tabelas 4 e 5. Cada SMC foi realizada com um número de cem mil amostras, e os piores casos foram considerados. O número de amostras foi determinado verificando-se a convergência dos resultados em relação aos erros admissíveis.

\section{Discussão}

Da metrologia, sabe-se que o erro global de uma medida pode ser decomposto em uma parcela determinística e outra aleatória. A primeira, também denominada erro sistemático, é passível de correção, já que pode ser determinada pelo processo de calibração.
A segunda, denominada erro aleatório, ou ainda, incerteza da medição, não pode ser corrigida, uma vez que seu valor varia aleatoriamente a cada medição. Levando em conta esse pressuposto na análise dos resultados de calibração (Tabela 3 ), há dois pontos a considerar:

1) Os erros relativos máximos apresentados pelos diversos subsistemas de medição, e mais expressivamente nos de nível de ruído e concentração de oxigênio, apontam para a necessidade de correções. Neste aspecto, a estrutura microcontrolada facilita a programação de funções de correção que podem ser modeladas a partir dos pontos de calibração; 

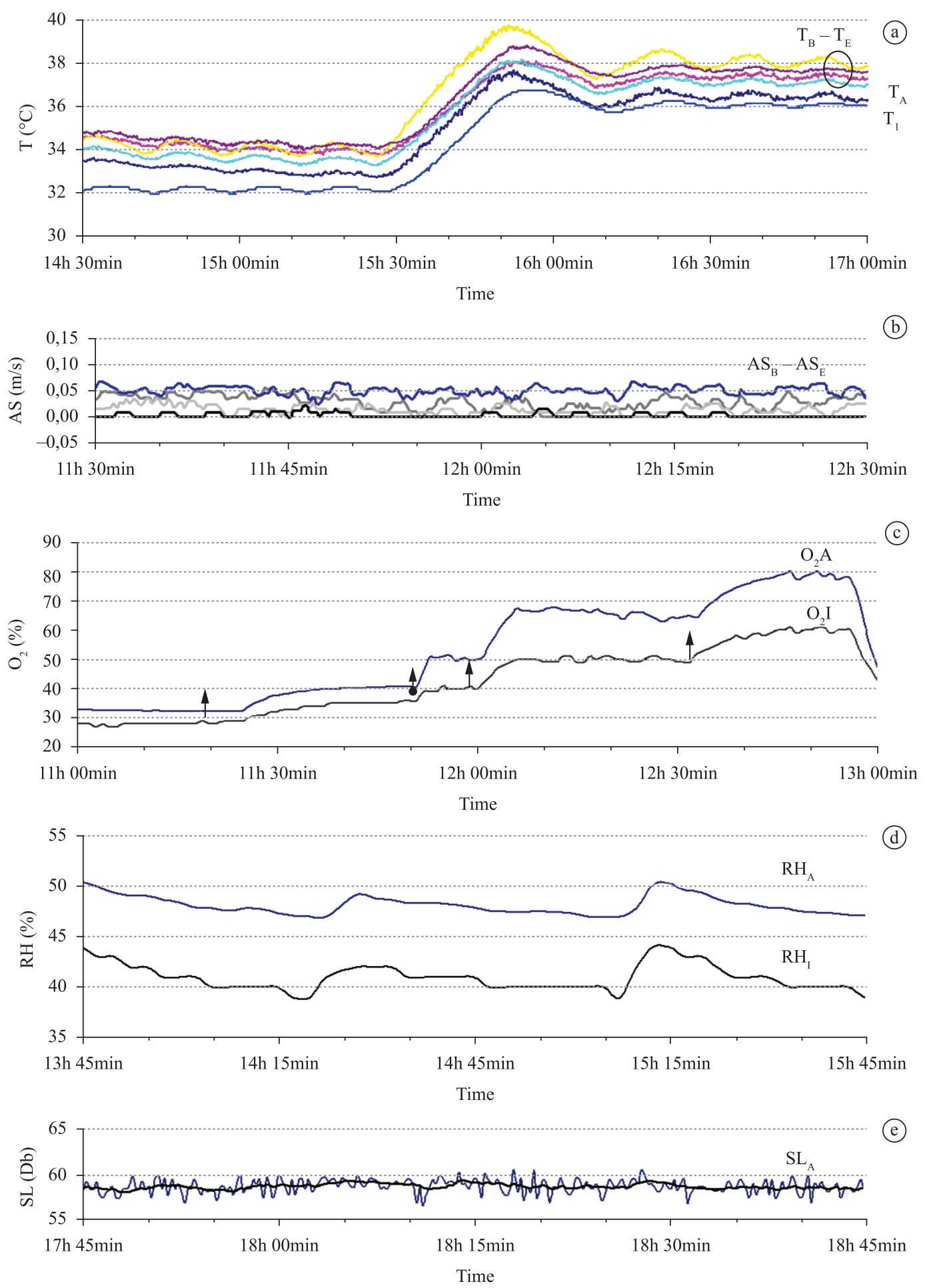

Figura 3. Exemplo de medições realizadas com o protótipo. a) Temperatura; b) Velocidade do ar; c) Concentração de $\mathrm{O}_{2}$; d) Umidade relativa e e) Nível sonoro. O subscrito I refere-se a valores indicados pela incubadora. Os subscritos A e B-E referem-se a valores indicados pelo analisador com os sensores posicionados no bloco central e nos periféricos, respectivamente.

Figure 3. Example of measurements performed with the prototype. a) Temperature; b) Air velocity; c) $O$, concentration; d) Relative Humidity and e) sound level. The subscript 'I'refers to the values indicated by the incubator. The subscripts 'A' and 'B-E' refer to the values given by the analyzer with sensors positioned in the central and peripheral blocks, respectively. 
ENSAIO 4: Gradientes de Temperatura da Incubadora

Resultado do Ensaio (Repetição: 6):

Temperatura média (A; B; C;D;E) $=(36,39 ; 37,67 ; 37,32 ; 37,99 ; 37,04){ }^{\circ} \mathrm{C}$

Gradientes $(\mathrm{B} ; \mathrm{C} ; \mathrm{D} ; \mathrm{E})=(1,28 ; 0,93 ; 1,60 ; 0,65){ }^{\circ} \mathrm{C}$

Referência: $+/-0,80{ }^{\circ} \mathrm{C}$

Conformidade: Não conforme a NBR IEC 60.601-2-19/50.102

ENSAIO 5: Variação da Temperatura da Incubadora

Resu7tado do Ensaio (Repetição: 2):

Temperatura da incubadora (média; máxima; mínima) $=(36,39 ; 36,88 ; 35,89){ }^{\circ} \mathrm{C}$

variações da temperatura em torno da média $=(+0,49 ;-0,50){ }^{\circ} \mathrm{C}$

Referência: $+/-0,50{ }^{\circ} \mathrm{C}$

Conformidade: conforme a NBR IEC 60.601-2-19/50.101

ENSAIO 6: Erro da Temperatura Indicada pela Incubadora

Resultado do Ensaio (Repeticão: 1):

Temperatura média da incubadora (Incubadora; Padrão) $=(32,00 ; 33,09){ }^{\circ} \mathrm{C}$

Erro: - 1,09 ${ }^{\circ} \mathrm{C}$

Referência: $+/-0,80{ }^{\circ} \mathrm{C}$

Conformidade: Não conforme a NBR IEC 60.601-2-19/50.106

ENSAIO 8: Sobre elevação e Tempo para Nova Estabilização

Resultado do Ensaio (Repetição: 1):

Sobre elevação da temperatura: $1,68{ }^{\circ} \mathrm{C}$

Tempo para nova estabi11 zação: 17,00 minutos

Referência (Sobre elevação; Tempo) $=(2,00 ; 15,00)$

conformi dade: Não conforme a NBR IEC 60.601-2-19/50.109

Figura 4. Partes do relatório de conformidade emitido pelo software de automação.

Figure 4. Parts of the compliance report issued by the automation software.

2) À exceção dos subsistemas de medição da concentração de oxigênio e velocidade do ar, as incertezas estimadas são compatíveis com os requisitos metrológicos definidos no projeto (Tabela 1). Com relação à concentração de oxigênio, observa-se que a própria incerteza da medição do padrão de calibração (Tabela 2) é superior à incerteza requerida para este subsistema. Embora essa calibração não possa corroborar os requisitos metrológicos do projeto e nem as especificações do fabricante do sensor, serviu para observar o comportamento do circuito e a sua tendência em relação a um equipamento calibrado. Idêntica situação também foi observada para o subsistema de velocidade do ar.

Do ponto de vista construtivo o protótipo apresenta diversas vantagens em relação aos analisadores comerciais existentes, que facilitam os ensaios de avaliação metrológica. A separação física entre o dispositivo móvel que contém os sensores e os circuitos de medição evita a influência da atmosfera da incubadora sobre os dispositivos eletrônicos utilizados nos circuitos de medição. A existência de sensores em quantidade idêntica aos pontos definidos pela norma NBR IEC 60601-2-19 e alocados sobre barras móveis agiliza o processo de avaliação, pois apenas um ajuste prévio na posição dos sensores é necessário para a realização de todos os ensaios. Desta forma é possível reduzir o tempo total de um ensaio, de $15 \mathrm{~h}$ para $7 \mathrm{~h}$ e $30 \mathrm{~min}$.
Tabela 4. Estimativa da incerteza para verificação da permutabilidade do subsistema de medição de temperatura.

Table 4. Uncertainty estimation for verification of the temperature measurement subsystem interchangeability.

\begin{tabular}{ccccc}
\hline \multirow{2}{*}{$\begin{array}{c}\text { Frequência/ } \\
\text { HZ }\end{array}$} & $\begin{array}{c}\mathbf{R}(\mathbf{T}) \\
\text { nominal/ } \Omega\end{array}$ & \multicolumn{2}{c}{$\begin{array}{c}\text { Temperatura } \\
\text { medida/ }\end{array}$} \\
\cline { 3 - 5 } & & Média & & $\mathbf{U}^{1}$ \\
\hline \multirow{2}{*}{0,13} & $12.493,3$ & 19,99633 & \pm & $0,17932^{2}$ \\
& $5.325,0$ & 39,99396 & \pm & $0,30360^{2}$ \\
\hline \multirow{2}{*}{0,000013} & $12.493,3$ & 19,99946 & \pm & $0,17934^{2}$ \\
& $5.325,0$ & 39,99932 & \pm & $0,30366^{2}$ \\
& $5.325,0$ & 39,99856 & \pm & $0,03745^{3}$ \\
\hline
\end{tabular}

${ }^{1}$ para $95 \%$ de confiança e $k=2 ;{ }^{2}$ com tolerância dos resistores, termistor e capacitor de $1 \%, 0,5 \%$ e $5 \% ;{ }^{3} \mathrm{com}$ tolerância dos resistores, termistor e capacitor de $0,02 \%, 0,3 \%$ e $5 \%$.

Com relação às medições com as incubadoras foram observados alguns pontos de não conformidade normativa importantes. A diferença das médias das temperaturas (gradiente) entre os blocos periféricos e o bloco central chegou a alcançar o dobro do valor máximo tolerado $\left(0,8{ }^{\circ} \mathrm{C}\right)$. $\mathrm{O}$ maior erro da temperatura indicada pela incubadora foi de $1,09^{\circ} \mathrm{C}$, para um limite máximo de $0,8^{\circ} \mathrm{C}$. O tempo para uma nova estabilização da temperatura foi de $17 \mathrm{~min}$., quando não deveria exceder $15 \mathrm{~min}$. $\mathrm{O}$ erro da concentração de oxigênio indicada pela incubadora foi crescente de 4,7 a $18,4 \% \mathrm{O}_{2}$, para uma faixa de 32,6 a 78,5\% $\mathrm{O}_{2}$ indicada pelo analisador. Este também excedeu o limite de 3,32 a $4,46 \% \mathrm{O}_{2}(2,5 \%+2,5 \%$ da leitura) estabelecido pela norma ISO 21647. Para 
Tabela 5. Estimativa da incerteza para verificação da permutabilidade do subsistema de medição do nível de ruído. Table 5. Uncertainty estimation for verification of the interchangeability of the noise level measurement subsystem.

\begin{tabular}{ccccc}
\hline Bloco & Variável & Média $(\overline{\mathbf{X}})$ & $\boldsymbol{u}_{\text {SMC1 }}$ & $\boldsymbol{u}_{\text {SMC2 }}$ \\
\hline $\begin{array}{c}\text { 1 } \\
\text { Microfone e }\end{array}$ & $V_{\mathrm{o}}$ & $948 \mathrm{mV}$ & $0,4 \%$ & $0,2 \%$ \\
amplificador & $D_{1}$ & $80 \mathrm{~dB}$ & $0,2 \mathrm{~dB}$ & $0,1 \mathrm{~dB}$ \\
\hline $\boldsymbol{2}$ & $R$ & $0,00 \mathrm{~dB}$ & $0,47 \mathrm{~dB}$ & $0,23 \mathrm{~dB}$ \\
Filtro & $C$ & Valor nominal & $1 \% / 4,37$ & - \\
ponderado & $D_{2(10 \mathrm{~Hz})}$ & Valor nominal & $5 \% / 4,37$ & - \\
tipo A & $D_{2(1 \mathrm{kHz})}$ & $0,00 \mathrm{~dB}$ & $0,17 \mathrm{~dB}$ & $0,29 \mathrm{~dB}$ \\
& $D_{2(20 \mathrm{kHz})}$ & $3,46 \mathrm{~dB}$ & $0,03 \mathrm{~dB}$ & $0,24 \mathrm{~dB}$ \\
1 e 2 & $D_{12(10 \mathrm{~Hz})}$ & $4,15 \mathrm{~dB}$ & $0,08 \mathrm{~dB}$ & $0,25 \mathrm{~dB}$ \\
\hline
\end{tabular}

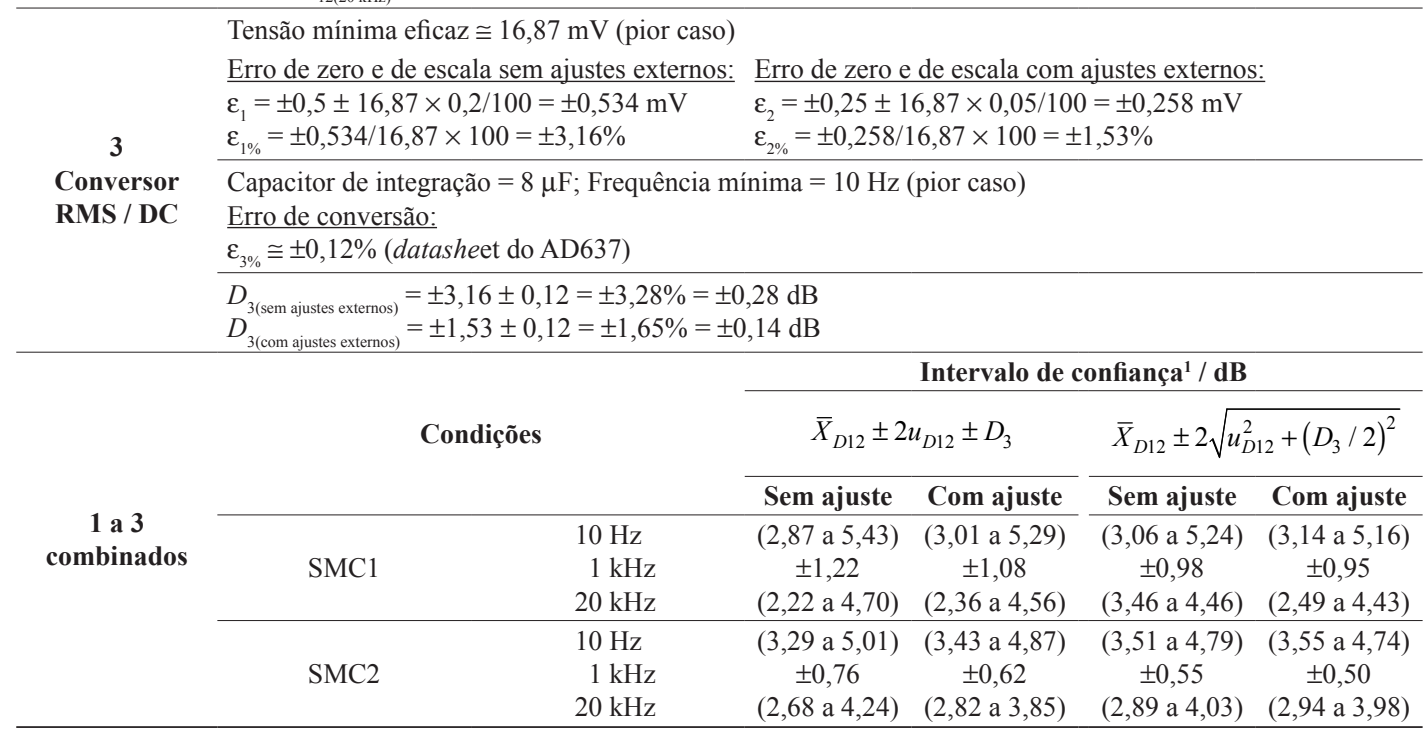

${ }^{1}$ A tolerância admitida pela norma IEC $61672-1$ é de $(-\infty$ a $5,5 \mathrm{~dB})$ para $10 \mathrm{~Hz}, \pm 1,4 \mathrm{~dB}$ para $1 \mathrm{kHz}$ e $(-\infty$ a $6,0 \mathrm{~dB})$ para $20 \mathrm{kHz}$.

a umidade relativa foi encontrado um erro constante de $7,1 \%$ UR para os valores indicados pela incubadora, excedendo $10 \%$ da leitura ou $4,8 \%$ UR, conforme define a norma NBR IEC 60601-2-19.

Devido à grande discrepância das medições da concentração de oxigênio, uma verificação da célula de $\mathrm{O}_{2}$ do protótipo foi realizada a $21 \%$ (concentração do ar ambiente) e a $99 \%$, com o auxílio de um cilindro com concentração de $99 \pm 0,21 \%$, certificado pelo INMETRO. Foi encontrado um erro máximo de $0,5 \% \mathrm{O}_{2}$.

Com relação ao nível de ruído e a velocidade do ar, ambos mantiveram-se abaixo do limite de tolerância de $60 \mathrm{~dB}$ e $0,35 \mathrm{~m} / \mathrm{s}$, respectivamente.

Da análise de incertezas para verificação da permutabilidade do subsistema de temperatura (Tabela 4) é possível observar que o subsistema não atende a esse requisito. Os piores casos foram identificados no extremo superior da faixa de medição $\left(40{ }^{\circ} \mathrm{C}\right)$ e praticamente não há influência da frequência do sinal de temperatura na incerteza expandida. É possível observar também que para obter a permutabilidade do subsistema é necessário reduzir as tolerâncias do sensor de temperatura e do resistor $R_{1}$, como indicado na terceira nota da Tabela 4 .

Quanto ao subsistema de medição do nível de ruído, o microfone é o único elemento que não é intercambiável, pois exige um ajuste devido à grande margem de tolerância de sua sensibilidade. Da análise efetuada (Tabela 5), foi possível identificar que esse é um elemento crítico e que sua incerteza padrão depende da qualidade metrológica dos instrumentos utilizados no processo de ajuste, como demonstrado pela incerteza padrão $u$ do desvio $D_{1}$, nas duas simulações SMC1 e SMC2. As simulações relativas ao filtro ponderado tipo A indicaram um desvio maior nos extremos da faixa de frequência, notoriamente em $10 \mathrm{~Hz}$, com média de $4,15 \mathrm{~dB}$ e incerteza padrão de $0,17 \mathrm{~dB}$. As simulações dos dois primeiros blocos juntos $\left(D_{12}\right)$ 
apresentaram média e incerteza padrão máximas em $10 \mathrm{~Hz}$, com média de $4,15 \mathrm{~dB}$ e incerteza padrão de $0,50 \mathrm{~dB}$ e $0,29 \mathrm{~dB}$, respectivamente aos dois ajustes de compensação do microfone. No terceiro bloco, as maiores incertezas foram devidas ao erro de zero e de escala, com $3,16 \%$ e $1,53 \%$, sem e com ajustes externos (trimpots), respectivamente. A incerteza devido à conversão foi bem menor, $0,12 \%$, em $10 \mathrm{~Hz}$. A incerteza deste bloco $\left(D_{3}\right)$, foi de $0,28 \mathrm{~dB}$ e $0,14 \mathrm{~dB}$, sem e com ajustes externos, respectivamente. Para frequências maiores que $10 \mathrm{~Hz}$, há uma tênue melhora deste resultado, pois apenas a menor parcela, relativa à conversão, depende (inversamente) da frequência. O intervalo de confiança resultante deste subsistema foi, no pior caso, de (2,87 a 5,43 dB). Uma redução mais significativa $(3,29$ a $5,01 \mathrm{~dB})$ foi obtida para o segundo caso de ajuste do microfone (SMC2), que admitiu o uso de instrumentos com incertezas menores, enquanto o ajuste de zero e de escala do conversor RMS/DC demonstrou menor impacto. Outra melhoria foi observada na segunda forma de estimativa deste intervalo, entretanto, menos segura, por desconhecer-se a FDP dos erros informados no datasheet do AD637. Todos os intervalos de confiança estimados para esse subsistema atenderam aos limites de tolerância prescritos pela a norma IEC 61672-1, conforme nota 1 da Tabela 5 .

\section{Conclusão}

Foi apresentado o desenvolvimento de um analisador de incubadoras neonatais com capacidade para medir, no microclima interno de incubadoras, temperatura, em cinco pontos distintos, velocidade do ar, em quatro pontos distintos, umidade relativa, concentração de oxigênio e nível de ruído. O protótipo também é capaz de se comunicar com um computador para transmitir dados de medição e receber comandos para a realização de ajustes remotos. O protótipo foi projetado para atender a requisitos de qualidade metrológica (Tabela 1) em acordo com normas técnicas preestabelecidas. Os resultados das calibrações efetuadas nos diversos subsistemas do protótipo (Tabela 3) atestam a confiabilidade do analisador embora haja a necessidade de correções de erros sistemáticos nas medições. Por dificuldades técnicas locais, as calibrações/comparações nos subsistemas de medição da velocidade do ar e da concentração de oxigênio não foram ideais, entretanto, mesmo com as inadequações, forneceram bons indícios de confiabilidade.

Desta forma, o analisador ora apresentado constituise em uma alternativa confiável e economicamente viável, possuindo ainda características de usabilidade e de comunicação que facilitam a automatização de ensaios de desempenho em incubadoras neonatais.

Destaca-se a necessidade de calibrações adequadas nos subsistemas de velocidade do ar e de concentração de oxigênio para completar a validação dos requisitos metrológicos do analisador. E ainda, a possibilidade de uso da metodologia apresentada, no desenvolvimento de outros protótipos analisadores de equipamentos médicos, contribuindo para a produção de analisadores nacionais de boa qualidade e baixo custo.

As não conformidades apresentadas nas medições que exemplificam a utilização do analisador reforçam a necessidade de monitoramento dos equipamentos em uso, facilitando a identificação de importantes fontes de risco para pacientes e usuários. É sugerido um trabalho investigativo que avalie um número significativo de incubadoras e identifique as principais fontes de erros das grandezas indicadas, contribuindo para o aprimoramento destes equipamentos.

As análises de incerteza da medição para verificação da permutabilidade dos componentes dos subsistemas de medição de temperatura e de nível de ruído apontaram para a necessidade de uma melhor adequação nas tolerâncias de sensores e elementos passivos, de forma que outras unidades possam ser produzidas sem a necessidade de ajustes. No caso do microfone de eletreto, não foi encontrado um dispositivo com margem de tolerância suficientemente reduzida para garantir a sua permutabilidade. Observa-se que tal ajuste é bastante comum nos modelos comercias desse tipo de medidor. Embora a permutabilidade seja uma característica desejável, a sua falta não inviabiliza a confiabilidade do analisador, considerando a necessidade de calibração individual de todos os subsistemas, por se tratar de um instrumento padrão, e da possibilidade de programação de rotinas para diminuição da incerteza nos pontos apontados nos procedimentos de calibração.

\section{Referências}

Ashton N, Ward B, Serpell G. Role of oxygen in the genesis of retrolental fibroplasia: a preliminary report. British Journal of Ophthalmology. 1953; 37(9):513-20. PMid:13081949. PMCid:1324188. http://dx.doi.org/10.1136/bjo.37.9.513

Associação Brasileira de Normas Técnicas - ABNT. NBR IEC 60601-2-19: Equipamento Eletromédico, Parte 2-19: Prescrições particulares para segurança de incubadoras para recém-nascidos $(\mathrm{RN})$. Rio de Janeiro: Associação Brasileira de Normas Técnicas; 2000.

Bercini LO. Mortalidade neonatal de residentes em localidade urbana da região sul do Brasil. Revista de Saúde Pública. 1994; 28(1):38-45. http://dx.doi.org/10.1590/ S0034-89101994000100005 
Carvalho AP, Pereira LF. Noise in infant incubators and in neonatal intensive care units. In: International Conference on Noise Control Engineering: Proceedings of the 1998 International Conference on Noise Control Engineering [Internet]; 1998 November 16-18; Christchurch, New Zealand. [cited 2010 mar 22]. Available from: http://paginas. fe.up.pt/ carvalho/in98.pdf

Falk SA, Farmer JC. Incubator noise and possible deafness. Archives of Otolaryngology - Head \& Neck Surgery. 1973; 97(5):385-7.

Howe WG. Two-sided tolerance limits for normal populations - Some improvements. Journal of the American Statistical Association. 1969; 64(326):610-20. http://dx.doi. org/10.2307/2283644

International Electrotechnical Commission - IEC. IEC 61672-1: Electroacoustics - Sound level meters Part 1: Specifications. Geneva: International Electrotechnical Commission; 2002. 85 p.

International Organization for Standardization - ISO. ISO 7767: Oxygen monitors for monitoring patient breathing mixtures - Safety requirements. Geneva: International Organization for Standardization; 1997.

International Organization for Standardization - ISO. ISO 21647: Medical electrical equipment - Particular requirements for the basic safety and essential performance of respiratory gas monitors. Geneva: International Organization for Standardization; 2004.

Kim YH, Kwon CH, Yoo SC. Experimental and numerical studies on convective heat transfer in a neonatal incubator. Medical \& Biological Engineering \& Computing. 2002; 40(1):114-21. PMid:11954698. http://dx.doi.org/10.1007/BF02347704

Maluf N, Williams K. An introduction to microelectromechanical systems engineering. 2th ed. Norwood: Artech House Publishers; 2004. 304 p.

Rego MAS, Anchieta LM. Assistência hospitalar ao neonato. Belo Horizonte: Secretaria de Estado de Saúde de Minas Gerais; 2005 [Internet]. [cited 2010 mar 25]. Available from: http://www.saude.mg.gov.br/publicacoes/linha-guia/ protocolos/assistencia_hospitalar_neonato.pdf

Rodrigues RG, Oliveira ICS. Os primórdios da assistência aos recém-nascidos no exterior e no Brasil: perspectivas para o saber de enfermagem na neonatologia (1870-1903). Revista Eletrônica de Enfermagem [Internet]. 2004 [cited 2011 nov 10]. Available from: http://www.revistas.ufg.br/index. php/fen/article/view/809/924

Steinhart JS, Hart SR. Calibration curves for thermistors. Deep Sea Research and Oceanographic Abstracts. 1968; 15(4):497-503. http://dx.doi. org/10.1016/0011-7471(68)90057-0

\footnotetext{
Autores

Josemir da Cruz Alexandrino

Programa de Pós-graduação em Engenharia Industrial, Universidade Federal da Bahia - UFBA, Rua Aristides Novis, $n^{\circ} 2,6^{\circ}$ Andar - Federação, CEP 40210-630, Salvador, BA, Brasil;

Núcleo de Tecnologia em Saúde - NTS, Instituto Federal de Educação, Ciência e Tecnologia da Bahia - IFBA,

Rua Emídio dos Santos, s/n, Barbalho, CEP 40301-015, Salvador, BA, Brasil
}

\section{Handerson Jorge Dourado Leite}

Núcleo de Tecnologia em Saúde - NTS, Instituto Federal de Educação, Ciência e Tecnologia da Bahia - IFBA, Rua Emídio dos Santos, s/n, Barbalho, CEP 40301-015, Salvador, BA, Brasil

\section{Alexandre Henrique Hermini}

Centro de Atenção Integral à Saúde da Mulher - CAISM, Universidade Estadual de Campinas - UNICAMP, Av. Alexander Fleming, 101, CEP 13083-881, Campinas, SP, Brasil

\section{Ricardo de Araújo Kalid, Herman Augusto Lepikson}

Programa de Pós-graduação em Engenharia Industrial, Universidade Federal da Bahia - UFBA,

Rua Aristides Novis, $n^{\circ}$ 2, $6^{\circ}$ Andar - Federação, CEP 40210-630, Salvador, BA, Brasil 